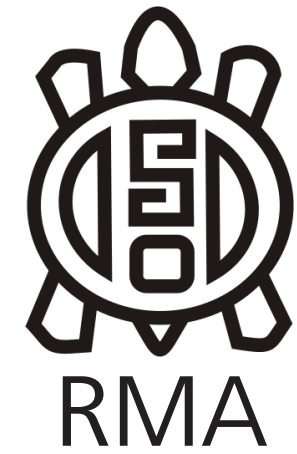

Arqueología

\title{
Las vegas de altura del Valle de El Bolsón (Dpto. Belén, Catamarca) y su articulación a los territorios locales
}

\author{
Highland marshes in El Bolsón Valley (Dpto. Belén, Catamarca) and its \\ articulation with local territories
}

\begin{abstract}
Marcos N. Quesada* Enrique A. Moreno*y Ana Soledad Meléndez*
*Centro de Investigación y Transferencia de Catamarca (CITCA), CONICET/ UNCA y Escuela de Arqueología, UNCA. Prado N³66 (4700).

San Fernando del Valle de Catamarca, Catamarca. mkesada@yahoo.com.ar; enalmor@gmail.com; solemelendez@gmail.com
\end{abstract}

\begin{abstract}
Resumen
Recientes prospecciones en las cabeceras de las quebradas de Los Nacimientos, Lampacillo y Yerba Buena, en el valle de El Bolsón, provincia de Catamarca, aportaron relevantes datos relacionadas con ocupaciones pastoriles, correspondientes posiblemente al siglo XIX y XX, así como también a poblaciones cazadoras-recolectoras representadas por fragmentos de puntas de proyectil, principalmente de formas lanceoladas, características del Arcaico y del período de transición hacia sociedades productoras de alimentos. Es llamativa a su vez la escasa presencia de evidencias correspondientes a los períodos Formativo y de Desarrollos Regionales, que son abundantes, en cambio, en el fondo del valle y en sectores intermedios de las quebradas laterales. Nuestro objetivo en este trabajo es presentar los resultados de estas prospecciones, así como también el avance del análisis de los materiales recuperados para evaluar los modos de articulación de las vegas de altura con los paisajes conocidos en sectores más bajos en distintos momentos de la historia de ocupación del valle.
\end{abstract}

Palabras clave: Territorios campesinos; Valle de El Bolsón; vegas de altura.

\begin{abstract}
Recent surveys in the head of Los Nacimientos, Lampacillo and Yerba Buena ravines contributed with relevant information about herding occupation, corresponding occupations possibly to the XIXth and the XXth century, as well as to hunter-gatherer groups represented by fragments of projectile point, principally of lanceolate designs, typical of the archaic and the transitional period towards peasants societies. It is remarkable the scanty presence of evidences corresponding to the Formative and Regional Developments periods, which are abundant, on the other hand, at the lower part of the valley and in intermediate sectors of lateral ravines. Our aim in this work is to present the results of these surveys, as well as advance on the analysis of the materials recovered. Thereby, to evaluate the way marshes places in highest parts articulates with the landscapes known in lower sectors in different moments of the history of occupation of the valley.
\end{abstract}

Keywords: Peaseants territories; Valle de El Bolsón; High altitude marshes.

\section{Las preguntas}

Distintas investigaciones enfocadas en el estudio del campesinado indígena de la puna en las últimas décadas han reconocido la variabilidad ambiental de esta región - antes era más bien considerada homogéneamente inhóspita - y han permitido la formulación de modelos para comprender modos específicos de explotación y apropiación de sus variados recursos (Haber 2006; Moreno 2010; Olivera 1991; Yacobaccio et al. 1997-98, entre otros). Al respecto, la arqueología, pero con mayor detalle la etnografía, han caracterizado un sistema de movilidad y asentamiento que explica la articulación de distintos microambientes en esquemas territoriales que involucran la dispersión en el espacio y el tiempo de distintas actividades productivas y/o extractivas. Por ejemplo, Delfino (2001) planteó, en base a un estudio etnoarqueológico de la territorialidad de unidades domésticas de Laguna Blanca (Catamarca, Argentina), un modelo según el cual un tipo de asentamiento permanente - las bases residenciales- se articula en el ciclo anual con otros, de carácter temporario, denominados puestos. A estos se le suma una tercera categoría -los paravientos- destinados a permanencias 
aún menos prolongadas que la de los puestos. Otros investigadores se han interesado en esta particular espacialidad y se ha alcanzado cierta coincidencia en que la relación bases residenciales - puestos podrían caracterizar la espacialidad doméstica actual y/o reciente en la puna y circumpuna, aunque con diferentes denominaciones según el caso y con variantes en cuanto al tiempo de permanencia, escala espacial y estructura de los ciclos temporales. En un esquema similar, Göbel (2002) describió paisajes pastoriles organizados mediante la articulación de "casas de campo" (conjuntos arquitectónicos semejantes a las bases residenciales) y "estancias" (conjuntos arquitectónicos semejantes a los puestos), como principales elementos de estructuraciones territoriales en el área de Susques (Jujuy, Argentina). Tomasi (2011) caracterizó, también para el área de Susques y Rinconada un modelo en el cual se articulan tres tipos de asentamientos: "casas en el pueblo", "casas de campo o domicilios" y "puestos o estancias". En el Zenta (cordillera oriental de Jujuy), Cladera (2013) propone la existencia de un modo de territorialidad fundado en la explotación de cuatro pisos ecológicos, según las denominaciones locales en - jujuy, valle, monte y cerro -, que implica para cada uno de ellos, distintos modos de instalación que se activan a lo largo del ciclo anual de trashumancia: viviendas con rastrojos, estancias y puestos.

Bastarán estos ejemplos para mostrar que, si bien con diferencias no menores en función de las ecologías locales, tiempos de ocupación, división sexual y etaria del trabajo, entre muchos otros factores, estos modelos podrían ser conceptualizados, en términos más generales, como un modo de territorialidad de escala doméstica conformado por un número de asentamientos que funcionan como unidad y son habitados por un grupo familiar o parte del grupo simultáneamente o de manera alternada en el ciclo anual, en una lógica espaciotemporal, que Tomasi (2011) llamó "multiplicidad del espacio doméstico".

Ahora bien, aunque estas formas de territorialidad parecen tener una amplia dispersión, aún se busca comprender su antigüedad y continuidad histórica. Göbel (2002) indicó un origen relativamente reciente, dentro del período republicano, para las "casas de campo" y las "estancias". Aunque, más recientemente, Yacobaccio et al. (2011) en base a la interpretación de los depósitos de Cueva Quispe (Jujuy), han remontado esta forma de estructuración del territorio al menos hasta el 1100 AP Por su parte Olivera (1991) ha caracterizado un modelo de asentamiento para las poblaciones del Formativo en Antofagasta de la Sierra (Catamarca, Argentina), que ha denominado "sedentarismo dinámico", vinculado directamente al tiempo de permanencia y acceso a recursos, en base a la caracterización ecológica local.

Cabe señalar las marcadas similitudes estructurales entre esos modelos de territorialidad propuestos tanto para los últimos dos milenios como para las familias campesinas actuales, que podrían ser indicativas de su gran antigüedad. Sin embargo, no hay motivos aún para sostener que su implantación haya sido igualmente temprana en toda la región y, tampoco, que una vez establecido se haya mantenido en el tiempo.

Específicamente para el área donde se desarrolla nuestra investigación, el valle de El Bolsón (Figura 1), se ha indicado la existencia de un modelo de territorialidad similar al que venimos mencionando, consistente en viviendas ubicadas en el fondo de valle que se articulaban con puestos dedicados principalmente, aunque no exclusivamente, a actividades ganaderas, desarrolladas en distintas alturas en laderas del valle (Kostanje 2005; Molina Pico 2015). En la actualidad estos puestos están, en su mayoría, deshabitados o con una intensidad de uso muy disminuida. Se trata de un proceso de abandono que parece haberse iniciado, o al menos acentuado, en las décadas del 1970 o 1980 (Molina Pico 2015).

Concretamente, los interrogantes que inspiraron y guiaron nuestra investigación pueden formularse como sigue: ¿Pueden los modelos conocidos de estructuración territorial para la puna y borde de puna, que hemos englobado bajo la expresión "multiplicidad del espacio doméstico", dar cuenta de las relaciones entre los asentamientos conocidos del piedemonte y fondo de

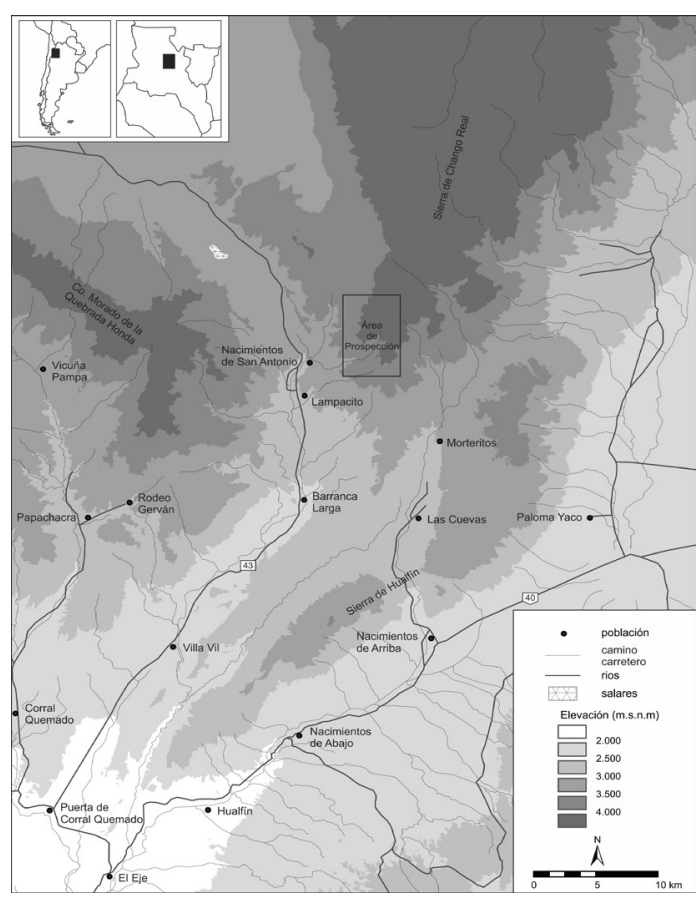

Figura 1. Cartografía del valle de El Bolsón. Se indica con un rectángulo el área de vegas de altura prospectada.

Figure 1. Cartography of the El Bolsón valley. The high vegas prospected area is indicated with a rectangle. 
valle con los de las tierras altas en el valle de El Bolsón? ¿Cómo estaban y están planteadas estas relaciones en este espacio geográfico? ¿Qué transformaciones sufrieron a través del tiempo y en relación a los cambios sociopolíticos que vivieron las poblaciones del oeste catamarqueño? Para ello es necesario, antes de continuar, recorrer brevemente las investigaciones arqueológicas y los resultados obtenidos en el valle.

\section{Antecedentes: paisajes arqueológicos del valle de El Bolsón}

El valle de El Bolsón, al oeste de la provincia de Catamarca, (Ver figura 1) se ubica en una posición intermedia entre los ambientes más definidos de Puna y valles bajos del NOA. Las investigaciones arqueológicas conducidas por A. Korstanje y su equipo, a partir de la década de 1990, centradas principalmente en sectores intermedios y el fondo de valle, han proporcionado un panorama de ocupaciones sucesivas que representan diversos momentos de la historia locales.

Existen pocas evidencias que ubican el inicio de la ocupación del valle desde el Holoceno Temprano. Entre ellas contamos con el hallazgo de una punta cola de pescado en la quebrada de El Alto El Bolsón (Korstanje 2005). Por otro lado, es frecuente el hallazgo en las quebradas laterales de diversas formas de puntas lanceoladas, cuyos diseños se vincularían con ocupaciones ocurridas durante el Holoceno Medio. Sin embargo, estos hallazgos superficiales son los únicos registros disponibles para ese dilatado rango temporal.

Desde el inicio del primer milenio de la era, en cambio, se produce una marcada transformación del paisaje, principalmente en los sectores bajos de las quebradas laterales, más frecuentemente en la porción norte del valle Korstanje (2005). Corresponden a este momento diversos conjuntos aldeanos vinculados a espacios agrícolas con arquitectura permanente. La Mesada/ Morro Relincho y El Alto El Bolsón/Yerba Buena son dos casos bien documentados, entre otros sitios detectados (Korstanje 2007; Quesada y Korstanje 2010; Quesada y Maloberti 2012). Korstanje propuso que estos sitios se articulaban, además, con otros como Cueva Pintada y los componentes formativos del Alero Los Viscos, destinados a ocupaciones discontinuas, principalmente aprovechando abrigos rocosos en los sectores sur y medio del valle, en proximidades de áreas de vegetación de monte con recursos para la recolección. Diversos sitios con arte rupestre ubicados principalmente en vías de tránsito que llevan a espacios de producción agrícola o hacia fuera del valle (Korstanje y Aschero 1998) y sitios que pudieron haber estado vinculados al tráfico caravanero, como El Médano, integraban los paisajes sociales durante el primer milenio d.C. (Korstanje 1998)

Algunas aldeas agrícolas del Formativo continuaron ocupadas durante el Período de Desarrollos Regionales (Quiroga y Korstanje 2013). Al mismo tiempo los sectores medio y sur del valle, en el fondo de cuenca ven la aparición de pequeñas aldeas que mantienen su vinculación de proximidad con espacios agrícolas (Quiroga 2005). Resulta claro, entonces, que fue durante el primer milenio y parte del segundo milenio de la era cuando se producen las transformaciones más marcadas en el paisaje local.

No disponemos hasta el momento de ningún registro contemporáneo al período Inka, mientras que el Colonial Temprano está apenas representado en el componente Hispano-Indígena del sitio Los Viscos. En alguna medida es posible que la baja representación del Colonial Temprano se deba a las dificultades que aún existen para reconocer las materialidades de ese período y distinguirlas de momentos anteriores y posteriores como bien ha señalado Quiroga (2005). Esta misma autora propone que en el período Colonial posterior al Hispano-Indígena se inició un proceso de desagregación que continuó a lo largo del periodo Republicano, en el cual se "abandonó el patrón aldeano prehispánico por la dispersión de pequeñas unidades de viviendas y corrales que señalan una clara tendencia hacia la reducción de la variabilidad y envergadura de la infraestructura productiva agrícola prehispánica" (Quiroga 2005:102). En las últimas décadas, en cambio, por motivos diversos, seguramente concurrentes $y$ aún poco estudiados, como la creciente influencia del empleo público, la escolarización, etc., se ha producido un nuevo fenómeno de concentración poblacional en unos pocos núcleos, en el fondo del valle de El Bolsón, como es el caso de Barranca Larga.

Reconociendo las transformaciones y continuidades que la organización espacial de la vida en el valle de El Bolsón sufrió a lo largo de esta dilatada historia vale preguntarse de qué modo las vegas de altura se vincularon a los diversos paisajes que venimos describiendo sucintamente.

\section{Las vegas de altura del Chango Real}

La prospección se concentró en la porción norte del valle de El Bolsón, en quebradas laterales que, en sus mayores cotas (4610 msnm) presentan características de la Puna. Geográficamente, el sector de trabajo comprende el área del faldeo sur del cerro Loma Corral, en las estribaciones meridionales de la Sierra del Chango Real (ver figura 1). El relieve allí se encuentra dominado por extensos y abruptos planos inclinados de derrubios que descienden hacia los colectores principales. Son excepciones a lo dicho las áreas seleccionadas para la prospección. Un análisis preliminar mediante imágenes satelitales y modelos digitales del terreno mostró que la quebrada de La Cueva, donde se ubican las vegas homónimas y la cuenca de la vega de Barro Negro se ubicaban en 
relieves algo más moderados, aunque de ningún modo regulares (Figura 2). Estimamos que, además del factor de localización que suponen las vegas que albergan estos espacios, el relieve menos abrupto podría haber propiciado una mayor conservación de eventuales sitios arqueológicos y facilitar la transitabilidad durante la prospección. Las tres expectativas indicadas fueron luego confirmadas en el terreno. La vega de Relincho, en cambio, está ubicada en un sector de fuerte pendiente e inicialmente no estaba incluida entre los objetivos de prospección. Sin embargo, como se encontraba en la ruta de ascenso desde nuestro campamento arqueológico en El Alto El Bolsón, fue incorporada a la muestra.

Relincho corresponde a la cuenca alta del río de El Alto El Bolsón, las vegas de la quebrada de La Cueva, a la cuenca alta del río de Lampacillo y la vega de Barro Negro a la cuenca alta del río de Los Nacimientos de San Antonio (ver figura 2). Allí se ubican los principales manantiales que alimentan esos cursos de agua. Hay al menos dos recursos en estos sectores que creemos pudieron haber sido atractivos en distintos momentos de la historia local: pastos palatables y vicuñas (Vicugna vicugna). Con respecto al primero, cabe consignar que en general se trata de un sector de vegas relativamente discontinuo constituido por manchones pequeños agrupados en torno a los cursos de agua. Tal es el caso de la quebrada de La Cueva donde la vega está fragmentada en, al menos, 40 espacios verdes de dimensiones variables (ver figura 2). Pese a esta fragmentación, en conjunto representan 6,64 ha de pastos de vega. Las de Relincho suman 2,21 ha mientras que la vega de Barro Negro, que sí es continua, agrega 8,32 ha. Debe señalarse que además de las mencionadas existen en las inmediaciones, dentro del radio de una jornada, una infinidad de otras vegas que, si bien se hallan en las áreas abruptas de difícil instalación y tránsito, pueden ser, de todos modos, aprovechadas por animales domésticos y silvestres. En total, entonces, la superficie de vega existente en el área suma aproximadamente 56,49 ha. Se trata de una extensión nada despreciable de pastos de calidad. Pero además de ello debe considerarse que se trata de uno de los sectores del valle que recibe la mayor tasa de precipitaciones orográficas (Korstanje 2005). También existe una disponibilidad estacional de "pastos del campo", es decir pasturas de importancia para el cuidado de la hacienda. Si bien actualmente no hay cría de llama en las vegas de altura de la región, sí la hubo en tiempos recientes. En cambio, sí hemos visto vacunos y equinos pastando en las vegas.

Las vegas de altura son uno de los pocos lugares del valle habitados actualmente por vicuñas. Las hemos visto descender al amanecer hacia las vegas y el lugar está completamente atravesado de sendas huelleadas por vicuñas. Además, registramos en el sector bosteaderos es decir, lugares recurrentes de defecaciones que marcan los límites del territorio de grupos familiares de vicuñas

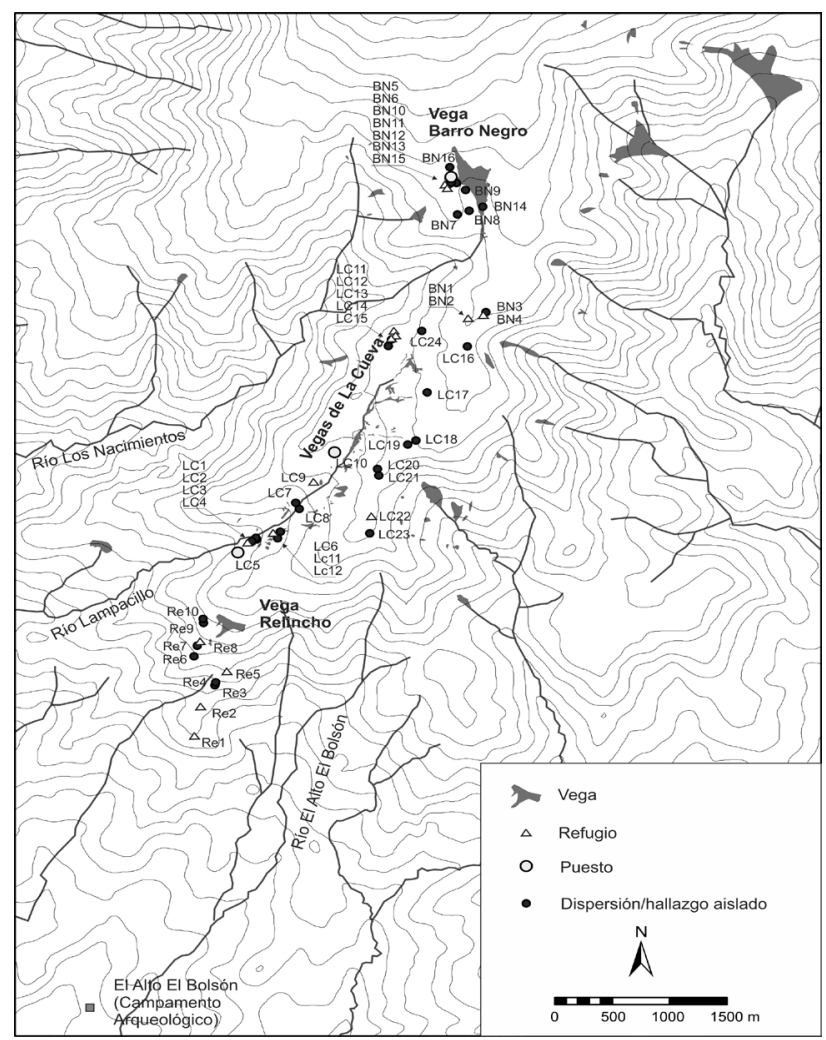

Figura 2. Área de prospección. Se indica la ubicación de los refugios, puestos y hallazgos aislados/dispersiones detectados en la prospección.

Figure 2. Survey area. The location of shelters, positions and isolated findings / dispersions detected in the survey are indicated.

(Wheeler 2006). Actualmente, la vega de Barro Negro está incluida en el territorio de grupos familiares de vicuñas, en relación con esto, observamos bosteaderos ubicados a menor cota, los que alcanzan el espacio que separa Barro Negro de las vegas de La Cueva. No conocemos si los territorios de las vicuñas incluían estas últimas vegas en el pasado, pero es probable que sí, incluso podrían haber llegado hasta las vegas de Relincho, como lo sugiere el nombre del lugar'.

Investigaciones paleoambientales recientes ofrecen una aproximación de los cambios ocurridos en el paisaje local durante el Holoceno Tardío a partir de diferentes proxies, (polen, geomorfología, sedimentos). La secuencia polínica de un testigo sedimentario extraído de Laguna Cotagua en el sector central del valle indicó que, durante los 6400 años A.P., abarcados por los depósitos del testigo, no se registraron variaciones en la composición de la vegetación, pero si se observan oscilaciones vinculadas a la cobertura vegetal, la que se caracterizó por una co-dominancia entre estepa herbácea y estepa arbustiva (Meléndez et al. 2018). Desde el inicio de la secuencia (6400 cal A.P.), extendiéndose hasta ca.

\footnotetext{
${ }^{1}$ Relincho es el término que se emplea en la puna catamarqueña para hacer referencia al macho alfa de un grupo familiar de vicuñas.
} 
2080 años cal A.P., habría ocurrido el periodo de mayor humedad en el valle, lo que es relativamente coincidente con lo registrado en áreas vecinas (Grana 2012, Oxman 2015, Ratto et al. 2013). La sedimentología del testigo, por el contrario, dio cuenta de cambios más abruptos en la tasa de sedimentación, con puntos de inflexión marcando momentos de agradación de fondos de valle, vinculados a cambios de la estabilidad geomorfológica en las laderas (mayor acumulación en los sectores bajos como consecuencia de mayor erosión de laderas) (Meléndez et al. 2018). Prospecciones geoarqueológicas en todo el valle, verificaron en las terrazas fluviales del río El Bolsón el proceso de agradación mencionado y luego, un proceso de incisión marcada, que ocurrió en paralelo a un avance de arenas de la Puna, que forman grandes dunas al norte del valle cubriendo algunos causes. Los procesos más recientes de intensificación de la actividad geomorfológica son registrados a partir del 1000 A.P., se caracterizaron por una degradación extendida de las laderas y los fondos de valle, reflejados en deslizamientos, erosión de suelos, torrencialidad e incisión dominante, entre otros. Eventos que fueron registrados en áreas vecinas, para los mismos momentos. Regionalmente se señala ca. 1000 años A.P. el inicio de un proceso de aridización, cuando los valles del NOA se encontraban ocupados por poblaciones agro-pastoriles (Brunotte et al. 1988, Grana 2012, Lupo, 1998, Lupo et al. 2016, Kulemeyer 2013, Oxman 2015, Sampietro Vattuone y Peña Monné 2016). El impacto generado

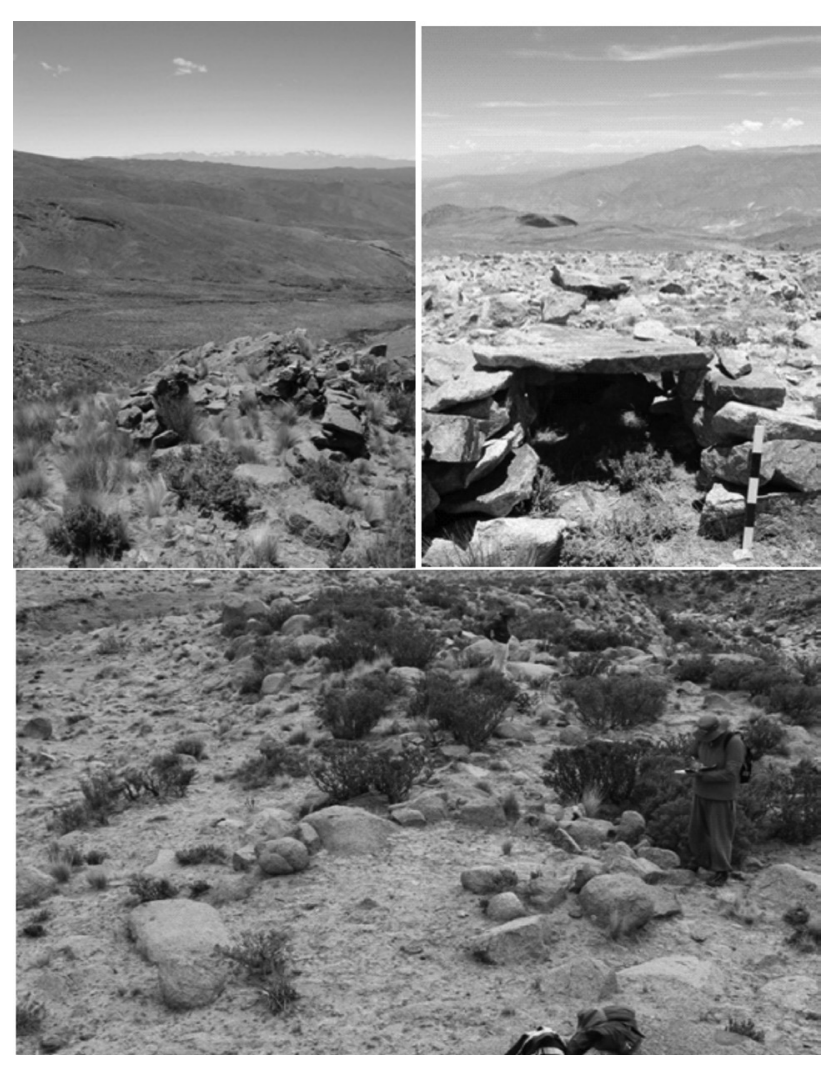

Figura 3. Ejemplos de refugios relevados durante la prospección.

Figure 3. Examples of shelters registered during survey. sobre el ambiente por las poblaciones y su relación con estos eventos entendidos como de degradación ambiental es un tema que se encuentra actualmente bajo estudio. En el caso particular de las vegas de alturas, los trabajos geoarqueológicos no registraron procesos erosivos importantes, lo que las ubica como el único espacio del valle que se mantuvo en condiciones de estabilidad ambiental durante los últimos 6400 años. Algunas cárcavas de poca profundad pueden indicar procesos erosivos incipientes vinculados al pastoreo de ganado vacuno actual (Meléndez et al. 2018).

\section{Resultados de la prospección}

Vamos a distinguir dos tipos de unidades de registro. Por un lado, las fijas, que comprenden estructuras arquitectónicas y ciertos factores de localización como cuevas o aleros, y móviles, que incluyen objetos aislados, en agrupaciones discretas (dispersiones) o asociados a estructuras. Estos últimos consisten en ítems de material lítico, fragmentos cerámicos y elementos de hierro como herraduras y latas de conserva.

Un total de 53 unidades de registro fueron identificadas de las cuales, 18 son estructuras y las restantes 35 son materiales recuperados aislados o en agrupaciones discretas en el paisaje (ver figura 2). Las estructuras refieren a dos lógicas constructivas claramente diferenciadas: puestos y refugios

\section{Refugios}

Los refugios $(n=15)$ consisten en pequeñas estructuras cerradas con diseños en planta de forma variable, que frecuentemente aprovechan de forma oportunista grandes rocas o aleros para completar el cerramiento. Un elemento distintivo de los refugios es que parecen haber estado destinados a estancias de muy corto término, no pudiéndose distinguir un interés por mejorar las condiciones de confortabilidad. Los tamaños suelen ser muy pequeños, la mayoría abarca entre 1 y $2 \mathrm{~m}^{2}$. La construcción suele ser bastante expeditiva tratándose siempre de pirca seca con mampuestos poco seleccionados y traba poco elaborada que no alcanza a detener completamente el viento ni aseguran un efecto térmico en su interior (Figura 3). Sólo en un caso una parte del recinto estaba provista de una precaria techumbre de piedras que alcanzaba a cubrir un pequeño sector del refugio. Por otra parte, los refugios no se encuentran equipados para tareas de reproducción cotidiana (fogón, enseres para la preparación y consumo de alimentos) y, sólo en algunos casos se localizan en proximidad del agua. La ubicación de los refugios es muy variable. Algunos se hallan vinculados a sendas en laderas y filos en pendiente, algunos se encuentran en proximidad de las vegas mientras que otros se ubican en sectores elevados, divisorias de agua en las cabeceras de quebradas o dorsales topográficas con excelente visión 
a uno y otro lado. No está claro que pueda asignarse una función particular a esta categoría de estructura. Aunque no están equipadas para ninguna actividad en particular podrían haber servido a una variedad de ellas, ya sea como puntos de observación de presas, refugios para pastores, descanso en un recorrido, o alternativamente todas ellas y otras que no imaginamos por el momento. En tres casos aparece material arqueológico vinculado a los refugios, específicamente desechos de talla y, en un caso, un instrumento. Un refugio contenía huesos de camélidos calcinados y en bastante mal estado de conservación ${ }^{2}$.

\section{Puestos}

De este tipo de estructura registramos 3 casos: La Cueva 005, La Cueva 010 y Barro Negro 011 (ver figura 2). La Cueva 005 es el menos elaborado de los tres, consta de una única habitación de planta oval con una medida interior máxima de 2,5 m. El exterior del vano de acceso, orientado al este, está nivelado mediante el aterrazamiento de un muro bajo compuesto por una alineación de rocas. Una vara de álamo, hallada a pocos metros de la estructura, parece haber sido la viga cumbrera que apoyaba en los hastiales. En el piso interior del puesto, un depósito de carbón y cenizas manifiestan la existencia de un fogón.

\footnotetext{
${ }^{2}$ Solamente se pudo diferenciar un cuneiforme, mientras que sólo una astilla de hueso largo no se encontraba calcinada
}

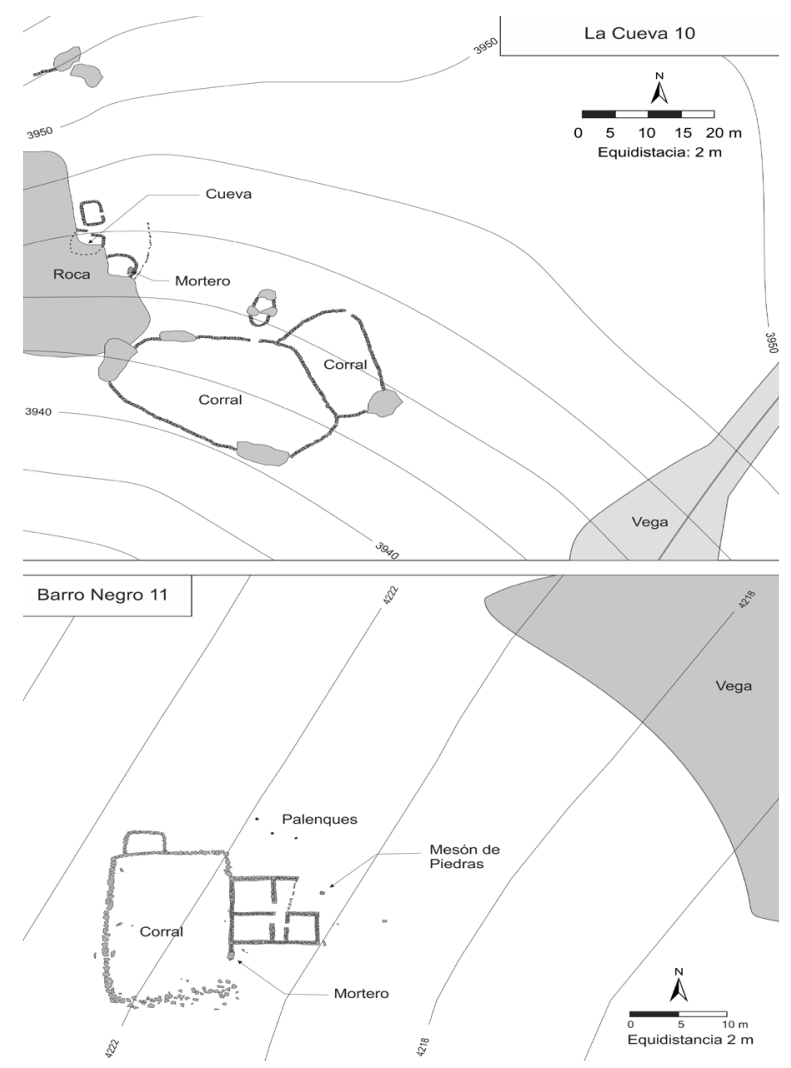

Figura 4: Planta de los puestos La Cueva 10 y Barro Negro 11.
Otro puesto, La Cueva 010, incluye tres recintos, dos de ellos construidos contra el gran afloramiento rocoso que se dispone al oeste del conjunto (Figura 4). De estos, el que se halla más al sur, completa el cerramiento con un muro bajo en forma de arco pronunciado. En su interior hay una piedra de moler plana y la mano que accionaba sobre ella. El otro recinto adosado al afloramiento logra el cerramiento mediante dos cortos muros que no alcanzan a unirse dejando entre ellos un vano de acceso. Este recinto fue construido en el preciso lugar donde el afloramiento forma un alero no muy profundo pero que brinda mayor protección. Dentro del recinto, bajo el alero, hay un depósito de cenizas, carbón y abundantes fragmentos de huesos calcinados que informa de un área de cocción y consumo de alimentos. Por último, el tercer recinto, de planta rectangular y muros más elevados fue construido separado del afloramiento y pudo haber sido un espacio de dormitorio. Una alineación de piedras que apenas sobresalen del suelo delimita una suerte de antepatio del conjunto arquitectónico y lo separa, por el sur, del área de corrales.

Hay cierta cantidad de material en superficie asociado a este puesto. Por un lado, recolectamos un conjunto de fragmentos cerámicos, que describiremos más adelante, y un conjunto de 11 especímenes líticos, de los cuales siete son desechos de talla y 4 instrumentos (una raedera, un perforador, una punta de proyectil y un cortante).

Por último, Barro Negro 011 es el tercer puesto relevado en la prospección (ver figura 4). Éste fue construido a pocos metros del borde de la vega de Barro Negro y, en particular, de uno de los tres ojos de agua o manantiales que la irrigan. Consta de tres recintos cuadrangulares adosados entre sí formando una $L$, a los cuales se accede desde un antepatio ubicado al este. Los muros, bien elaborados, rematan en hastiales que resolvían la techumbre a dos aguas. De las tres habitaciones, la del suroeste pudo haber sido una cocina, a juzgar por las cenizas depositadas allí, aunque también pudo haber sido reutilizada con ese fin en tiempos más recientes cuando comenzó a ser empleada como refugio. Frente al antepatio, hacia el este, hay un mesón de piedra como los empleados para llevar adelante el sacrificio de los animales y, hacia el norte, tres largas piedras bien clavadas en el suelo hacen las veces de palenques. A las tres habitaciones se les adosa, por el oeste, un gran corral, al cual se une, a su vez, un recinto más pequeño que pudo ser un chiquero. El ingreso al gran corral es por una abertura en el muro este, al sur de los recintos de habitación. Sobre uno de los muros, al norte del acceso, hay una gran piedra de moler plana con su mano móvil.

En el caso de este puesto, registramos dos desechos de talla (uno en basalto y uno en obsidiana) y tres instrumentos formatizados. Estos últimos se caracterizan por un cortante de basalto con retoque unifacial cuyo 
bisel activo se encuentra fracturado, una preforma de punta de proyectil de basalto y un fragmento apical de punta de proyectil, posiblemente de diseño lanceolado.

Generalizando, los puestos consisten en conjuntos arquitectónicos de relativa complejidad. Su organización espacial responde, por un lado, a la dinámica de manejo de la hacienda ya que aparecen vinculados a grandes corrales de pirca y/o próximos a vegas sobre las cuales tienen vistas privilegiadas. Por otro lado, resulta claro que, a diferencia de los refugios, los puestos han sido acondicionados para permanencias relativamente largas. La arquitectura, más formalizada, se realiza a partir de muros que están elaborados con piedras seleccionadas unidas con argamasa. Esto brinda mayor estabilidad para muros elevados (con la posibilidad de permanecer en pie en el interior de los recintos), mayor protección del viento y estabilidad térmica. En los tres casos registrados estaban presentes como rasgo arquitectónico hastiales para sostener techumbres a dos aguas. Además, se encuentran dotados de espacios y enseres orientados a la realización de actividades relacionadas con la reproducción cotidiana. Los tres casos muestran áreas de fogón, mientras que La Cueva 010 y Barro Negro 011 disponen de pesadas piedras para moler posiblemente grano o charqui. Por su parte, Barro Negro 011 dispone del mesón de piedra para beneficiar animales. Los puestos semejan casas y al parecer permanecían equipados con herramientas pues, al menos en Barro Negro 011, aún perdura allí una hoja de pala de hierro.

\section{Caracterización de los materiales}

\section{Líticos}

Durante la prospección realizada registramos 47ítems que corresponden a materiales líticos que presentan muy buen estado de conservación, prácticamente sin presencia de pátina o ventifacción de aristas, filos y negativos de tallado.

Uno de los principales aspectos que resalta de este conjunto es que, a pesar de ser relativamente reducido, presenta cierta diversidad en el uso de las materias primas, predominando el basalto con el $45 \%$ de representación en la muestra $(n=21)$, seguido del cuarzo (36\%, $n=17)$ y la obsidiana $(15 \%, n=7)$. La cuarcita y la dacita se encuentran caracterizadas sólo por un espécimen cada una. Con respecto a la obsidiana corresponde aclarar que el porcentaje expresado arriba incluye piezas provenientes de, al menos, dos fuentes de aprovisionamiento Laguna Cavi y Ona (Escola et al. 2009), situadas en la puna a más de $90 \mathrm{~km}$. de distancia.

En el conjunto registramos 31 desechos de talla y 16 instrumentos. Los primeros presentan un alto porcentaje de fracturas (75\%). La gran mayoría de los desechos corresponden a lascas angulares (70\%),
Tabla 1. Relación entre las distintas materias primas identificadas durante la prospección y su vinculación a clases tipológicas.

Table 1. Relationship between the different raw materials identified during the survey and their connection to typological classes.

\begin{tabular}{cccc}
\hline & Desecho & Instrumento & Total \\
\hline Basalto & 21 & 9 & 30 \\
Cuarcita & 0 & 1 & 1 \\
Cuarzo & 17 & 1 & 18 \\
Obsidiana & 7 & 4 & 11 \\
Dacita & 0 & 1 & 1 \\
\hline
\end{tabular}

observándose algunos desechos con reserva de corteza. Con respecto a los tamaños de los desechos, obtenemos una representación bastante equilibrada de los mismos, notándose un pico en los tamaños medianos. En conjunto, esta información nos refiere a una técnica de talla basada en la percusión, la limpieza de nódulos y la obtención de formas base aptas para la manufactura de instrumentos.

Los instrumentos muestran la manufactura de ocho puntas de proyectil y dos preformas de puntas de proyectil, una raedera, tres cortantes, un perforador y un bifaz de arista sinuosa.

En relación a las materias primas registramos nueve instrumentos manufacturados en basalto, cuatro en obsidiana, uno en cuarzo, uno en dacita y uno en cuarcita. Sin embargo, si evaluamos la representación de instrumentos en relación a la totalidad de materiales registrados, notamos que en el caso del basalto, prácticamente el $50 \%$ de la piezas corresponden a instrumentos, mientras que en obsidiana supera este valor. Por su lado, el cuarzo presenta solamente un instrumento manufacturado, un bifaz de muy simple manufactura. En el caso de la cuarcita y la dacita, los únicos especímenes registrados corresponden a instrumentos (Tabla 1).

En términos generales, llama la atención la relativa alta inversión de trabajo si consideramos que el $75 \%$ de los instrumentos presenta retoque bifacial, siendo principalmente marginal, aunque cuatro piezas presentan retoque parcialmente extendido y dos extendido.

La presencia de puntas de proyectil, son un aspecto relevante a tener en cuenta y profundizar en el futuro la investigación sobre paisajes de cacería en el valle.

Tal como comentamos más arriba, registramos ocho puntas de proyectil. Se trata de un conjunto en cual sobresale la obsidiana como la materia prima más utilizada para la manufactura de estos instrumentos $(n=4)$. Otras dos fueron manufacturadas en basalto $y$ las restantes en cuarcita y dacita. En este sentido, ya ha sido destacado, para otros sectores, la elección de la 
obsidiana para la manufactura de puntas de proyectil, quizá por sus características tecnológicas y/o estéticas, pese a la considerable distancia a las fuentes de aprovisionamiento (Chaparro 2009; Escola et al. 2009; Lazzari 1997; Moreno 2011; Yacobaccio et al 2004).

El análisis de estos instrumentos aporta información acerca de la profundidad histórica de la ocupación de las vegas altas del Chango Real, considerando el diseño interpretado. En este sentido, registramos dos especímenes que se vinculan posiblemente con momentos entre el inicio y fines del primer milenio. Uno de estos especímenes, manufacturado en obsidiana proveniente de Laguna Cavi, presenta limbo triangular y pedúnculo destacado puede ser comparado con el diseño PPC, propuesto por Hocsman (2006) para la microrregión de Antofagasta de la Sierra y que en otros sitios del NOA y del norte chileno han sido asignados al período correspondiente entre el 3000 y el 1800 AP (De Souza 2004, Moreno 2010, 2011). El otro espécimen, también manufacturado en obsidiana presenta limbo triangular, base convexa y es de pequeñas dimensiones, lo que suele vincularse con ocupaciones tardías e incluso incaicas (Escola 2000; Elías 2014; Hocsman 2006; De Souza 2004) (Figura 5 a y b).

Las otras puntas de proyectil recuperadas se encuentran fracturadas, registrándose sólo partes del limbo o ápices. Sin embargo, resulta claro que, por el espesor de los ápices o de los sectores del limbo presentes, se trata de diseños lanceolados comparables con ocupaciones más tempranas, desde aproximadamente el 4500 y el 3000 AP (Hocsman 2006, Martínez 2003, Moreno 2011) que las de las puntas de proyectil asignables al primer milenio y que se caracterizaron en el párrafo anterior.

\section{Cerámica}

Es llamativa la ausencia de material cerámico en

Tabla 2. Comparación de frecuencias de instrumentos y desechos de talla entre los conjuntos recuperados de la prospección en las vegas de altura y los materiales registrados en las partes bajas de la quebrada.

Table 2. Comparison of instrument frequencies and size debris between the assemblies recovered from prospecting in the highlands and the materials recorded in the lower parts of the creek.

\begin{tabular}{lcccc}
\hline & \multicolumn{2}{c}{ El Alto del Bolsón } & \multicolumn{2}{c}{ Vegas de altura } \\
& Desecho & Instrumento & Desecho & Instrumento \\
\hline Andesita & 0 & 1 & 0 & 0 \\
Basalto & 300 & 83 & 21 & 9 \\
Cuarcita & 31 & 9 & 0 & 1 \\
Cuarzo & 18 & 0 & 17 & 1 \\
Filita & 7 & 4 & 0 & 0 \\
Obsidiana & 35 & 2 & 7 & 4 \\
ND & 15 & 7 & 0 & 0 \\
Dacita & 0 & 0 & 0 & 1 \\
\hline Total & 406 & 106 & 45 & 16 \\
\hline
\end{tabular}

prácticamente todo el espacio prospectado, aspecto que contrasta fuertemente con lo observado en los sectores intermedios y fondo de valle donde de este tipo de material es mucho mayor (Puente 2010; Meléndez 2018; Sastre 2017). Se registraron solo dos conjuntos, uno de 24 fragmentos en La Cueva 010 y otro, compuesto por 28 fragmentos en La Cueva 016. En ambos casos los fragmentos fueron separados por Familias o Grupos de Fragmentos, división que responde a las características observables a simple vista como ser, los tipos de pastas y el acabado de superficies. (Orton et al. 1993). Se observo además el grado de erosión y alteración de bordes y superficies siguiendo los enfoques tafonómicos para material cerámico de Pérez Winter et al. (2010) y Sanhueza Riquelme (1998) que fueron aplicados antes, en conjuntos cerámicos recuperados al sur del área de estudio, en sitios ubicados en el fondo de valle (Meléndez y Sentinelli 2017).

El conjunto recuperado en La Cueva 010 se halla muy fragmentado con tamaños que van desde 1 a $3,5 \mathrm{~cm}$ de diámetro, tamaños que no permitieron establecer formas posibles. El conjunto corresponde al menos, a cinco familias o grupos de fragmentos con tipos de cocción tanto oxidante como reductora. Los bordes se encuentran redondeados y las superficies presentan diferentes grados de erosión y alteración. Uno de los fragmentos podría corresponder al estilo Belén negro sobre rojo identificado en otros sectores del valle (Puente 2010). El otro conjunto recuperado (LC 016), por las características en la pasta, podrían pertenecer a una única vasija. Si bien, las superficies de los fragmentos están muy alterados por su exposición al viento, algunos fragmentos se encuentran decorados y podrían ser asignados al estilo Santa María bicolor también presentes en sitios del fondo de valle (Puente 2010). Los tamaños de este conjunto no supera los $4 \mathrm{~cm}$ de diámetro, a excepción de uno de 5,6 centímetros que se corresponde a un fragmento de asa.

Además, se recuperaron tres piezas metálicas, asociadas a los puestos, que consisten en dos fragmentos de herraduras y una lata de picadillo.

\section{La temporalidad del paisaje en las vegas de altura}

El conjunto lítico recuperado durante la prospección permite introducir algunos datos e interpretaciones relevantes acerca de la modalidad e historia de uso de estas vegas de altura. El primer dato de interés deriva de la procedencia de las materias primas. Tomando el trabajo realizado por Somonte (2004) específicamente para el área, identificamos como materias primas de origen distante al basalto y obsidiana, que fueron utilizadas para la manufactura de instrumentos. Mientras tanto, se identificó en cuarzo sólo un instrumento en etapa de manufactura. Por lo tanto, a pesar de la abundancia local de cuarzo, esta materia prima se utilizaba en una 
baja proporción para la manufactura. Por el contrario, las puntas de proyectil manufacturadas en obsidiana presentan una alta representación de reactivación de los biseles, práctica relacionada con la prolongación del uso de los instrumentos.

Por otro lado, el basalto es aprovechado de manera más generalizada para la manufactura de instrumentos, principalmente de retoque bifacial, asociados con actividades de corte. En el caso de la cuarcita y la dacita, solamente fue aprovechada para la manufactura de dos puntas de proyectil, por lo que no contamos con mayor información para profundizar el análisis.

Entonces, si bien reducido, el conjunto lítico nos informa de una alta proporción de instrumentos formatizados, frente a los desechos de talla, lo que implicaría poca frecuencia de tareas de elaboración de instrumentos. Posiblemente en estos espacios elevados sólo se habrían realizado tareas de mantenimiento o reactivación de filos. Sólo en el caso del cuarzo es posible que se hayan realizado instancias de formatización de filos.

Las características del conjunto lítico de las vegas de altura contrastan con las del sitio El Alto del Bolsón, ubicado a menor altura (3200 msnm aproximadamente), donde a pesar de notarse la utilización de prácticamente las mismas materias primas, se observa una relación entre instrumentos (19\%) y desechos de talla (72\%) que se vincularía a la presencia de talleres líticos que incluye el registro de núcleos (Tabla 2) (Moreno 2017).

Por otro lado, se resalta la alta presencia porcentual de puntas de proyectil en relación al conjunto total de instrumentos de dicho conjunto $(62,5 \%, n=10)$. Esto se vincularía con la relevancia de las prácticas cinegéticas en este espacio. En el caso del conjunto de El Alto del Bolsón, las puntas de proyectil corresponden sólo al $20 \%$ del conjunto total analizado $(n=137)$.

En relación a la historia del uso de estos paisajes, el diseño de las puntas de proyectil otorga algunos datos preliminares. En este sentido, la mayor parte de las puntas de proyectil, se vinculan con momentos anteriores al primer milenio de la era, solamente dos piezas, presentan diseños y tamaños asignables a dicho período histórico. Esta información permite suponer una profundidad histórica importante para las ocupaciones de las vegas de altura, aunque los datos hasta el momento no nos permiten asignar con mayor precisión estos especímenes y por lo tanto el uso de estos espacios. Sin embargo, no es posible, por el momento, conocer si estos hallazgos se vinculaban temporalmente a otros componentes como los refugios, debido a que no disponemos de elementos para ubicar cronológicamente a estos últimos y no hay asociación directa entra las puntas de proyectil y refugios. No obstante, fácilmente podríamos imaginar que las actividades de cacería que representan los instrumentos líticos en cuestión pudieron incluir cierta permanencia en estas vegas elevadas y, por lo tanto, pudieron implicar la confección y uso de refugios, como en otros paisajes cinegéticos conocidos como por ejemplo en la quebrada de Antofalla o en la microrregión de Antofagasta de la Sierra (Aschero y Martínez 2001, Moreno 2010, 2012, 2013, Ratto 2003). Con respecto a las puntas de proyectil morfológicamente asignables al Formativo o a la transición hacia el formativo, si bien aparecen en cercanías de dos puestos, parece poco probable una vinculación cronológica ya que estos últimos aparentan tener un origen mucho más reciente. En cambio, sí resulta más factible que estas puntas puedan ser relacionadas a los mismos factores de localización que motivaron la construcción de los puestos en lugares precisos: el alero aprovechado para la construcción de la cocina de La Cueva 010 y la cercanía a los ojos de agua próximos a Barro Negro 011.

Por otra parte, es llamativa la ausencia de cerámica correspondiente al periodo Formativo, siendo en cambio un elemento conspicuo en los numerosos sitios ubicados en los sectores medios y bajos de las mismas quebradas. Al parecer, las actividades llevadas a cabo durante estos momentos en las vegas de altura no requerían del uso de recipientes cerámicos, quizá por ser ocupaciones que, aunque pudieron ser repetidas, correspondían a eventos de muy corta duración.

En cambio, los fragmentos de valor cronológico de los dos únicos contextos de hallazgo de cerámica corresponden al período de Desarrollos Regionales. Se trata del fragmento Belén negro sobre rojo hallado en La Cueva 10 y el conjunto de fragmentos Santa María bicolor (LC016), que aparecen de manera aislada en un roquedal. Posiblemente la cerámica hallada en La Cueva 10, al igual que la punta de proyectil de diseño triangular con pedúnculo y dimensiones pequeñas, pueda ser explicada por su vinculación al alero rocoso.

No disponemos de ningún dato de ocupaciones de estas vegas de altura durante el período inca ni para el colonial. En cambio, para momentos más recientes disponemos de numerosas evidencias de ocupaciones. Los tres puestos, aunque al parecer La Cueva 10 y Barro Negro 11 fueron erigidos sobre los restos de ocupaciones anteriores, parecen tener un origen que difícilmente se remonte más allá del siglo XIX. El estado de conservación -aún conservan argamasa y materiales constructivos de madera- así lo sugiere. Algunos elementos hallados en estos sitios podrían proporcionar más precisión. Por ejemplo, la hoja pala hallada en Barro Negro 11 corresponde a un tipo de pala con costura y remaches (ver figura 5) que eran adquiridas por compra o como parte del salario en los ingenios azucareros de Salta adonde, a partir de la década de 1920, los pobladores locales concurrían como fuerza de trabajo estacional 


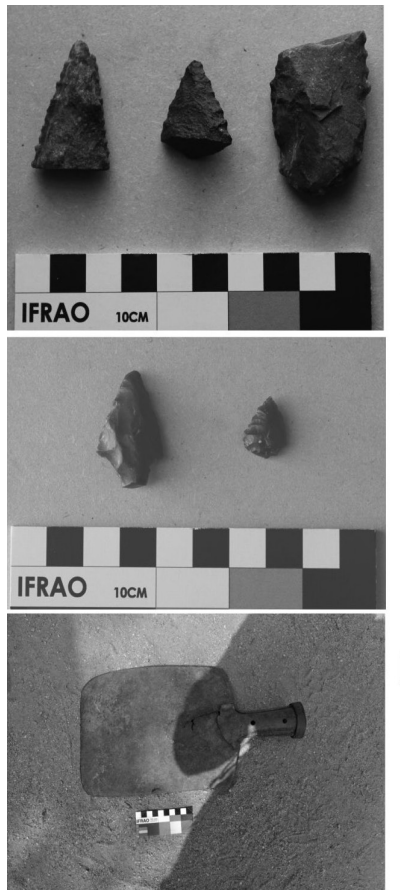

A

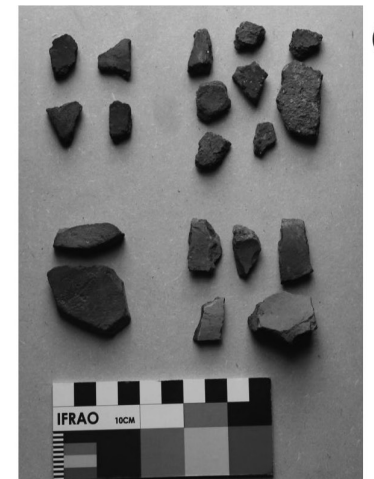

$E$

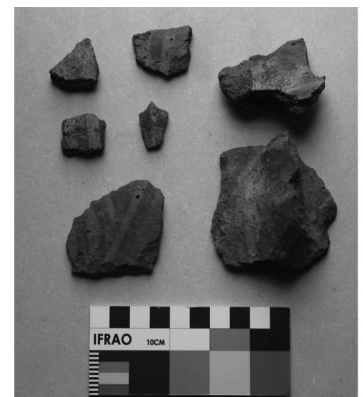

Figura 5: Hallazgos aislados o vinculados a estructuras. A) Puntas de proyectil asignadas a períodos de transición; B) puntas de proyectil correspondientes al formativo; C) y D) conjuntos de fragmentos cerámicos y E) hoja de pala.

Figure 5: Isolated findings or linked to structures. A) Projectile points assigned to transitional periods; B) Formative projectile points; C) and D) groups of ceramic fragments and E) shovel blade.

hasta que la mecanización de los emprendimientos redujo la demanda de mano de obra no especializada alrededor de 1960 o 1970, tal como es registrado en los trabajos de Gordillo (2010) y, específicamente para el valle por Molina Pico (2015). Otros elementos podrían corresponder a este mismo momento, como los fragmentos de herraduras y las sendas a la cuales se vinculan. Todos los puestos dejaron de funcionar como tales, las estructuras dejaron de ser mantenidas o reparadas y hoy están en ruinas, aunque ello no implica que hayan dejado ser usados. Latas de picadillo de hace sólo un par de años en Barro Negro 011 informan de su utilidad actual, como refugio, en estadías muy breves, posiblemente como un alto en el camino.

\section{Conclusiones}

Para concluir este trabajo, mas no la investigación, es conveniente en este punto retomar las preguntas que nos orientaron hasta aquí.

¿Pueden los modelos conocidos de estructuración territorial para la puna y borde de puna, que hemos englobado bajo la expresión "multiplicidad del espacio doméstico", dar cuenta de las relaciones entre los asentamientos conocidos del piedemonte y fondo de valle con los de las tierras altas en el valle de El Bolsón?
¿Cómo estaban y están planteadas estas relaciones en este espacio geográfico? ¿Qué transformaciones sufrieron a través del tiempo y en relación a los cambios sociopolíticos que vivieron las poblaciones del oeste catamarqueño?

Los registros obtenidos durante la prospección dan cuenta de una integración de las vegas de altura del Chango Real a los territorios de los grupos sociales que poblaron el valle desde momentos muy antiguos. A excepción de los períodos Inca y Colonial, el resto de la secuencia aparece representada. Lo que cambia, sin embargo, parece ser la intensidad y modalidad de ocupación de estos espacios. Si bien sostenida en el tiempo la ocupación de estas vegas altas no implicó permanencias prolongadas en el lugar durante la mayor parte del lapso temporal considerado. La evidencia recuperada no da cuenta de lugares acondicionados para tal efecto ni de utillaje requerido para la reproducción cotidiana de la fuerza de trabajo, como ollas, por ejemplo, excepto para el periodo de Desarrollos Regionales cuando sí fueron llevadas allí unas pocas, pero que conforman un registro que en absoluto alcanza a corresponderse con la intensidad con que, a la sazón, eran ocupados los sectores de fondo de valle y tramos medios de las quebradas laterales. Pareciera que, salvo un breve lapso durante el siglo XX (y quizá algo del XIX), la ocupación de las vegas de altura pueda caracterizarse como de una "modalidad refugio", siendo visitadas por breves momentos con el objetivo de realizar actividades específicas, principalmente cacería de vicuñas. En cambio, recién hacia mediados o fines del siglo XIX, se activaría la "modalidad puesto", es decir permanencias sostenidas en el lugar de duración al menos estacional, la cual parece ser entonces un fenómeno efímero y reciente. Ello indicaría entonces que, excepto ese breve y reciente periodo en que estuvieron activos los puestos, es poco probable que las vegas de altura que venimos estudiando fueran objeto de un modo de apropiación que pueda ser comprendido en el marco del modelo de la multiplicidad del espacio doméstico ya que la evidencia no da cuenta de permanencias sostenidas con arquitecturas acordes a esa modalidad de ocupación, sino más bien de estadías que, si bien pudieron ser recurrentes, eran de corta duración.

Por supuesto que las modalidades de apropiación de las vegas elevadas del Chango Real, que venidos caracterizando como "modalidad puesto" y "modalidad refugio", no deben ser vistas como antagónicas ni alternativas. Por el contrario, fácilmente puede advertirse que, aunque los refugios corresponden a episodios de muy corta duración, pudieron haberse integrado o

\footnotetext{
${ }^{3}$ Aunque Ingold refiere a una forma de tenencia (tenure) y no de territorialidad (territoriality). Antes que nosotros Cladera (2013) notó lo oportuno que resultaba la conceptualización de Ingold para caracterizar la territorialidad de la articulación puesto/estancia en el Zenta.
} 
articulado a la dinámica espacio-temporal centrada en los puestos cuando estos estuvieron activos.

Entonces, no es sólo la mayor intensidad de ocupación de los puestos, entendida en términos de mayor permanencia y diversidad de actividades, lo que distingue la modalidad "refugio" de la modalidad "puestos", sino también que, en virtud de aquella intensidad, la vida en los puestos es capaz de articular una forma de territorialidad cero-dimensional, para utilizar la denominación propuesta por Ingold ${ }^{3}$ (1987). Es así porque los puestos, que son casas, se constituyen como inicio y final de recorridos, itinerarios, desplazamientos que terminan configurando un espacio apropiado, un territorio, pero de límites difusos, permeables y móviles. La materialidad del puesto, visible y duradera, aparece y permanece en el paisaje como referente monumentalizado de esa apropiación. De hecho, los puestos abandonados no son demolidos $^{4}$, sino que permanecen como referentes de lugar, de ancestralidad -en ocasiones incluso adoptan los nombres de sus constructores- y pertenencia, aun cuando en gran medida los territorios que se articulaban a partir de estos ya han dejado de ser practicados.

No conocemos aún qué factores estimularon una mayor intensidad de ocupación de estas vegas de altura y tampoco cuáles el abandono de los puestos, aunque la coincidencia temporal con el periodo de migración estacional a los ingenios azucareros en el cual participaron muchas familias del valle (Molina Pico 2015), parecería esconder una relación relevante entre ambos procesos históricos. Una hipótesis al respecto es que, dado que el trabajo que requiere la práctica del pastoreo no tiende a concentrarse en un período o momento del ciclo anual, como en cambio sí sucede en la agricultura (Mayer 2004), es posible que las familias menguadas en número de miembros, y por lo tanto en su capacidad de movilizar fuerza de trabajo, hayan preferido, en tal condición, enfatizar en el componente pastoril de sus diversificadas economías campesinas intensificando, por lo tanto, el uso y ocupación de las zonas de producción más orientadas a esta actividad. Sin embargo, esta hipótesis permanece aún en un plano de conjetura y deberá ser explorada con mayor profundidad en nuestra agenda de investigación futura.

San Fernando del Valle de Catamarca, 5 de noviembre de 2018

\section{Agradecimientos}

Este trabajo fue realizado gracias al financiamiento de CONICET, ANPCyT y CIUNT. Mirella Lauricela y Gonzalo Moyano participaron de las prospecciones

\footnotetext{
4 Tal situación se vincula claramente al fenómeno de las "casas mochas" estudiadas en la vecina localidad de Laguna Blanca (Delfino 2015) y Puna jujeña (Rivet y Tomasi 2016) aunque nosotros no hemos registrado en Barranca Larga ese término para referir a los puestos deshabitados.
}

realizadas en las vegas altas del valle de El Bolsón. Queremos agradecer especialmente a los evaluadores de este trabajo, sus observaciones y comentarios han enriquecido notablemente el texto.

\section{Referencias citadas}

Abeledo, S. 2005. Los Pastores de la altura. Una mirada evolutiva de la vida en Atacama. Tesis de Licenciatura de la carrera de Ciencias Antropológicas. Facultad de Filosofía y Letras. Universidad de Buenos Aires.

Aschero, C y J. Martínez, 2001. Técnicas de caza en Antofagasta de la Sierra, Puna meridional, Argentina. Relaciones de la Sociedad Argentina de Antropología XXVI: 215-241.

Brunotte, E., Garleff, K., Stingl, H., 1988. Anthropogene Beeinflussung der Morphodynamik im Bolsón von Fiambalá / Nordwestargentinien. Abh. Akad. Wiss. Göttingen, Math.-Phys. Kl., III/41, 307-327.

Chaparro, M. G. 2009. El manejo de los recursos líticos en el pasado. Sociedades pre-estatales y estatales en el área valliserrana del Noroeste Argentino (1000-1450 d.c.). Tesis doctoral. Facultad de Filosofía y Letras, Universidad de Buenos Aires.

Cladera, J. 2013. Pessoas que Cruzam Territórios e Territórios que são Pessoas - As Experiências do Direito Espacial nos Andes: um estudo de caso no noroeste argentino. ILHA Revista de Antropología 15(1): 149-178. Universidade Federal de Santa Catarina.

Delfino, D. 2001. Of pircas and the limits of society: ethnoarchaeology in the la Puna, Laguna Blanca. Catamarca. Argentina. En Ethnoarchaeology of Andean South America: Contributions to Archaeological Method and Theory, editado por Lawrence A. Kuznar, pp. 116137. International Monographs in Prehistory, Ann Arbor, Michigan.

Delfino, D. 2015. Los vendavales de políticas estatales y el resistente lenguaje de las "casas mochas" en la puna catamarqueña. Cuadernos FHyCS-UNJu 47:181-195.

De Souza, P. 2004. Tecnologías de proyectil durante los períodos Arcaico y Formativo en el Loa Superior (Norte de Chile) a partir del análisis de puntas líticas. Chúngara. Volumen especial. I: 61-76.

Elías. A. 2014. Técnicas líticas diversas entre las sociedades de Antofagasta de la Sierra (Provincia de Catamarca, Puna Meridional Argentina) posteriores a ca. 1100 A.P. Estudios Atacameños 47: 59 - 82. San Pedro de Atacama.

Escola, P., M. Glascock, A. Korstanje y N. Sentinelli. 
2009. Laguna Cavi y El Medano. Obsidianas en circulación caravanera. $2^{\circ}$ Congreso Argentino y $1^{\circ}$ Congreso Latinoamericano de Arqueometría, pp: 103108. Comisión Nacional de Energía Atómica.

Grana, L. 2012. Arqueología y Paleoambiente: Dinámica cultural y cambio ambiental en sociedades complejas de la Puna meridional argentina. Tesis Doctoral. Universidad de Buenos Aires

Göbel, B. 2002. La arquitectura del pastoreo: Uso del espacio y sistema de asentamientos en la Puna de Atacama (Susques). Estudios Atacameños 23:53-79.

Gordillo, G. 2010. Lugares de diablos. Tensiones del espacio y la memoria. Prometeo. Bs. As.

Haber, A 2006. Una arqueología de los paisajes puneños. Domesticidad, interacción e identidad en Antofalla. Primer y segundo milenios d.C. Jorge Sarmiento Editor. Universitas Libros, Córdoba.

Hocsman, S. 2006. Producción lítica, variabilidad y cambio en Antofagasta de la Sierra -ca. 5500 - 1500 AP. Tesis doctoral inédita. Facultad de Ciencias Naturales y Museo. Universidad Nacional de La Plata.

Ingold, T. 1987. Territoriality and tenure: the appropriation of space in hunting and gathering societies. En The appropriation of nature. Essays on human ecology and social relations, editado por $\mathrm{T}$. Ingold, pp.130-164. University of Iowa Press, lowa City.

Korstanje, M. A. 1998. El Médano, ¿es un sitio caravanero? Apuntes sobre contextos de tráfico y territorialidad para el Formativo. En Los desarrollos locales y sus territorios, compilado por B Cremonte, pp. 33-64. CREA. Facultad de Humanidades y Cs. Sociales (UNJu) Jujuy.

Korstanje, A. 2005. La organización del trabajo en torno a la producción de alimentos en sociedades agropastoriles formativas. (Provincia de Catamarca, República Argentina). Tesis Doctoral inédita. Instituto de Arqueología y Museo, Facultd de Ciencias Natu rales e Instituto Miguel Lillo, Universidad Nacional de Tucumán. San Miguel de Tucumán.

Korstanje, M.A. 2007. Territorios Campesinos: Producción, Circulación y Consumo en los Valles Altos. En Procesos Sociales Prehispánicos en los Andes Meridionales, Editado por A. Nielsen, M.C. Rivolta, V. Seldes, M. Vazquez Y P. Mercolli. Vol. 2. Editorial Brujas

Korstanje, M. A. y C. Aschero. 1996. Arte Rupestre en los valles El Bolsón y Las Cuevas (Catamarca, Argentina): formulando hipótesis de cambio y conflicto. Chungara 28 (1 y 2): 199-222.
Korstanje, M. A. y P. Cuenya. 2008. Arqueología de la agricultura: suelos y microfósiles en campos de cultivo del Valle del Bolsón, Catamarca, Argentina. En Matices Interdisciplinarios en Estudios Fitolíticos y de otros Microfósiles, editado por A. Korstanje y P. Babot. BAR International Series 1870, pp. 133-147.

Kulemeyer, J.J., Lupo, L.C., Madozzo Jaén, C., Cruz, A., Cuenya, P. Maloberti, M., Cortés, G., Korstanje, M., 2013. Desarrollo del Paisaje Holoceno en la Cuenca de El Bolsón, Catamarca: gente y ambiente en procesos de cambio y estabilidad. Diálogo Andino 41: 25-44.

Lazzari, M. 1997. La economía más allá de la subsistencia: intercambio y producción lítica en el Aconquija. Arqueología 7:9-50. Facultad de Filosofía y Letras, Universidad de Buenos Aires.

Lupo, L.C. 1998. Estudio sobre la lluvia polínica actual y la evolución del paisaje a través de la vegetación durante el Holoceno en la cuenca del río Yavi. Borde Oriental de la Puna, Noroeste argentino. Tesis Doctoral. Universidad de Bamberg, Alemania.

Lupo L. C., Kulemeyer, J.J., Sánchez, A.C., Pereira, E.A., Cortes, G., 2016. Los archivos paleoambientales en el Borde Oriental de la Puna y sus respuestas a los cambios naturales y antrópicos durante el Holoceno. Noroeste argentino. Estudios Sociales del NOA. Instituto Interdisciplinario Tilcara - Filo: UBA. 16, 39-68.

Mayer, E. 2004. Casa, Chacra y Dinero. Economías Domésticas y Ecología en los Andes. IEP Ediciones, Lima.

Meléndez, A.S. y J. J. Kulemeyer. 2016. Lugares de Ocupación Humana y Evolución del Paisaje Holoceno Tardío en el Valle de El Bolsón (Dpto. Belén, Provincia de Catamarca). En Actas del XXI Congreso Nacional de Arqueología, pp. 555-558. San Miguel de Tucumán.

Meléndez, A.S. y Sentinelli, N. 2017. Dinámica ambiental y materiales arqueológicos de las terrazas fluviales del río El Bolsón (Dpto. Belén, Catamarca). Mundo de Antes 11: $99-127$.

Meléndez A.S., Kulemeyer, J.J., Lupo, L.C., Quesada, M.N. Korstanje M.A. 2018. Cambios en el paisaje en un valle del oeste catamarqueño durante el Holoceno Tardío. Integrando Arqueología, Palinología y Geomorfología. Arqueología 24(1) enero-abril: 31-51

Molina Pico, A. 2015. Prácticas de movilidad espacial en el Valle de El Bolsón, Depto. de Belén (Catamarca). Los tiempos de la zafra azucarera desde el presente de sus pobladores. Tesis de licenciatura inédita. Facultad de Filosofía y Letra, Universidad de Buenos Aires.

Moreno, E. 2010. Arqueología de la caza de vicuñas 
en el área del Salar de Antofalla, Puna de Atacama. Una aproximación desde la arqueología del paisaje. Tesis doctoral inédita. Facultad de Ciencias Naturales y Museo. Universidad Nacional de La Plata.

Moreno, E. 2011. Tecnología de caza en la quebrada de Antofalla. Revista del Museo de Antropología 4: 1732. Facultad de Filosofía y Humanidades, Universidad Nacional de Córdoba.

Moreno, E. 2012. Propiciando el encuentro. La estructuración de los paisajes de cacería en el contexto andino. Intersecciones en Antropología 13: 327-343, Facultad de Ciencias Sociales, UNICEN.

Moreno, E. 2013. Estrategias de caza y paisajes culturales en Antofagasta de la Sierra, Catamarca. Comechingonia 17(2): 95-121.

Moreno, E 2018. Tecnología lítica en el Valle de El Bolsón (Belén, Catamarca). Una primera aproximación a su caracterización. En preparación.

Olivera, D. 1991. El Formativo en Antofagasta de la Sierra (Puna meridional Argentina). Análisis de sus posibles relaciones con contextos arqueológicos agroalfareros tempranos del Noroeste Argentino y Norte de Chile. Actas de XI Congreso Nacional de Arqueología Chilena. Tomo II: 61-78. Museo Nacional de Historia Natural, Santiago de Chile.

Orton, C., P. Tyers y A. Vince. 1993. Pottery in Archaeology. Cambridge University Press, Cambridge.

Oxman, B. I., 2015. Paleoambiente y sociedad durante el Holoceno en la Puna de Jujuy: Un abordaje palinológico. Tesis Doctoral, Facultad de Filosofía y Letras, Universidad de Buenos Aires.

Puente, V. 2010. Prácticas de Producción Alfarera en el Valle del Bolsón (Belén, Catamarca). Materias Primas y Modos de Hacer CA. 900 - 1600 D.C. Tesis Doctoral. Universidad de Buenos Aires

Pérez Winter, C.; M. Fagundes y C. S. Rodrígues 2010. Una aproximación tafonómica al análisis arqueológico del material cerámico: caso experimental sitio São Lourenço 1, Municipio de Ituiutaba (MG). Geociências 9(1) pp.14-33.

Quesada, M. y M. A. Korstanje. 2010. Cruzando estructuras: El espacio productivo y su entorno percibido desde las prácticas cotidianas. En El hábitat prehispánico. Arqueología de la arquitectura y de la construcción del espacio organizado, editado por M. E. Albeck, M. C. Scattolin y M. A. Korstanje, pp. 119-147. Editorial Crivelli, Salta.
Quesada, M. y M. Maloberti. 2015. Continuidades en la construcción del paisaje agrario entre los Períodos Formativo y de Desarrollos Regionales en el oeste de Catamarca (siglos I a XV). En Racionalidad Campesinas en los Andes del Sur. Reflexiones sobre el Cultivo de la Quinoa y otros Vegetales Andinos, editado por P. Cruz, R, Joffre y Th. Winkel, pp: 140-165. EdiUju, S. S. de Jujuy.

Quiroga, L. 2003. El Valle del Bolsón (siglos XVII-XVIII). La Formación de un Paisaje Rural. Anales 6. Nueva Época. Local, Regional, Global: Prehistoria e Historia en los valles Calchaquíes: 301-327. University of Goteborg.

Quiroga, L. 2005. Disonancias en arqueología histórica: la experiencia del Valle del Bolsón. Revista Werken, segundo semestre, $\mathrm{n}^{\circ}$ 007. Universidad Internacional SEK, pp. 89-109. Santiago, Chile.

Quiroga, L., y M. A. Korstanje, 2013. Arqueología del campesinado en el valle del Bolsón. Producción y residencia como líneas de análisis para una escala de larga duración. En Uso de sistemas de información. geográfica en la arqueología sudamericana, editado por M. J. Figuerero Torres y A. D. Izeta, pp. 101-124. BAR, Oxford.

Ratto, N. 2003. Estrategias de caza y propiedades del registro arqueológico en la Puna de Chaschuil (Departamento de Tinogasta, Catamarca, Argentina). Tesis doctoral inédita. Facultad de Filosofía y Letras, Universidad de Buenos Aires.

Ratto, N., Montero, M.C., Hongn, F., Valero Garcés, B., 2013. La historia ambiental de las sociedades productivas del oeste catamarqueño (Catamarca), siglos I al XVI. N. Ratto (Comp.) Delineando prácticas de la gente del pasado: los procesos socio-históricos del oeste catamarqueño. Argentina, pp. 45-65.

Rivet, C. y J. Tomasi. 2016. Casitas y Casas Mochas. Los antiguos y los abuelos en sus arquitecturas (Coranzulí y Susques, provincia de Jujuy, Argentina). En Wak'as, diablos y muertos: alteridades significantes en el mundo andino, compilado por L. Bugallo y M. Vilca, pp. 373411. EdiUnju e IFEA, S.S. de Jujuy.

Tomasi, J. 2011. La casa como una construcción múltiple y colectiva. Aproximación al espacio doméstico en Susques y Rinconada. En Puna y arquitectura. Las formas locales de la construcción, editado por J. Tomasi y C. Rivet, pp: 41-52. CEDODAL, Buenos Aires.

Sampietro Vattuone M. M. y Peña Monné J.L. (Eds.) 2016. Geoarqueología de los Valles Calchaquíes. Universidad Nacional de Tucumán.

Sanhueza Riquelme, L.1998. Antecedentes y proposición metodológica para el estudio de huellas de alteración en la cerámica. Conserva 2:69-79. 
Sastre, Y. 2017. Producción, circulación y consumo alfarero. Aportes al estudio de la cerámica del valle de El Bolsón (Dpto. Belén, Catamarca) para el período Formativo ca. 900 a.C.- ca. 900 d.C. Tesis de Grado. Universidad Nacional de Tucumán.

Somonte, C. 2004. Informe Preliminar del Análisis TecnoMorfológico y Tipológico de los Conjuntos Líticos de la Microrregion del Valle del Bolson, Catamarca. Informe Agencia Nacional de Promoción Científica. Tucumán

Wheeler, J. 2006. Historia natural de la vicuña. En Investigación, conservación y manejo de vicuñas, editado por B. Vilá, pp: 25-36. Proyecto MACS. Universidad Nacional de Luján.

Yacobaccio, H., C. Madero, M. Malmierca y M. Reigadas.
1997-98. Caza, domesticación y pastoreo de camélidos en la Puna Argentina. Relaciones de la Sociedad Argentina de Antropología XXII-XXIII: 389-418.

Yacobaccio, H., P. Escola, F. Pereyra, M. Lazarri y M. Glascock. 2004. Quest for ancient routes: obsidian sourcing research in Northwestern Argentina. Journal of Archaeological Science 31: 193-204.

Yacobaccio, H., M. P. Catá, M. R. Morales, P. Solá, M. S. Alonso, M. Rosenbusch, C. Vázquez, C. T. Samec, B. Oxman y M. Cáceres. 2011. El uso de cuevas por pastores andinos: El caso de Cueva Quispe (Susques, Puna de Jujuy). En Arqueología de la Puna Argentina. Perspectivas actuales en el estudio de la diversidad y el cambio cultural, editado por G. E. López y H. J. Muscio, pp. 33-47. BAR, Archaeopress, Oxford. 